\title{
On the Nuclearity Condition for Massless Fields
}

D. BUCHHOLZ and P. JACOBI

II. Institut für Theoretische Physik, Universität Hamburg, D-2000 Hamburg 50, F.R.G.

(Received: 18 February 1987)

Abstract. It is shown that quantum field theories of free massless particles satisfy a recently proposed 'nuclearity condition' restricting the admissible number of local degrees of freedom of a theory. This result is based on an improved bound for the 'nuclearity index' of certain specific sets of vectors in Fock space.

\section{Introduction}

A new criterion restricting the admissible number of local degrees of freedom of a quantum field theory was recently proposed in [1]. This 'nuclearity condition' already has proved to be useful in the investigation of several structural problems in the general setting of quantum field theory [1-5]. It seems to be a natural basis for the discussion of questions involving considerations of phase space. We recall here in brief the relevant concepts.

DEFINITION. A subset $\mathcal{N}$ of a Hilbert space $\mathscr{H}$ is called a nuclear set if there exists a sequence of unit vectors $\Phi_{n} \in \mathscr{H}, n \in \mathbb{N}$ and of linear functionals $l_{n} \in \mathscr{H}^{*}, n \in \mathbb{N}$ such that

(i) $\sum_{n=1}^{\infty} \sup \left\{\left|l_{n}(\Psi)\right|: \Psi \in \mathcal{N}\right\}<\infty$,

(ii) $\sum_{n=1}^{\infty} l_{n}(\Psi) \cdot \Phi_{n}=\Psi \quad$ for all $\Psi \in \mathcal{N}$.

The nuclearity index $v(\mathcal{N})$ of $\mathcal{N}$ is defined by

$$
v(\mathscr{N})=\inf \sum_{n=1}^{\infty} \sup \left\{\left|l_{n}(\Psi)\right|: \Psi \in \mathscr{N}\right\}
$$

where the infimum is to be taken with respect to all vectors and functionals complying with the above conditions.

With the aid of these concepts, the nuclearity condition has been formulated in the algebraic setting of local quantum field theory as follows. (For an equivalent formulation, suggested by Longo, cf. [2].)

\section{CONDITION OF NUCLEARITY [1]}

Let $\mathfrak{x}(\mathcal{O})$ be the local von Neumann algebra of observables (resp. fields) associated with any bounded region $\mathcal{O}$ in Minkowski space, let $\Omega$ be the vector representing the vacuum, 
and let $H$ be the Hamiltonian. Then the subsets of vectors

$$
\mathcal{N}(\mathcal{O}, \beta)=\left\{\mathrm{e}^{-\beta H} U \Omega: U \in \mathfrak{U}(\mathcal{O}), U^{*} U=1\right\}
$$

in the physical Hilbert space $\mathscr{H}$ must be nuclear for any $\beta>0$. Moreover, there must exist positive constants $c, n$ and $r_{0}, \beta_{0}$ such that for all $\beta \leqslant \beta_{0}$ and $r \geqslant r_{0}$, where $r$ is the diameter of $\mathcal{O}$,

$$
v(\mathcal{N}(\mathcal{O}, \beta)) \leqslant \mathrm{e}^{c r^{3} \beta^{-n}} .
$$

The heuristic motivation for this criterion given in [1] is based on the assumption that, as far as the size of the sets $\mathcal{N}(\mathcal{O}, \beta)$ is concerned, one may ignore the fuzzy localization properties of the respective vectors and identify these sets with the canonical ensemble occupying a finite volume ('box') at temperature $\beta^{-1}$. The bound on the nuclearity index given above then derives from the expected behavior of the partition function in theories with a physically reasonable number of degrees of freedom. This qualitative picture was confirmed in [1] by explicit calculations in models on noninteracting massive particles.

It is the purpose of the present Letter to demonstrate that the nuclearity condition also holds in theories of noninteracting massless particles, such as free quantum electrodynamics. Moreover, it will be shown that one can put $n=3$ in the bound for the corresponding nuclearity index. So this quantity behaves for small $\beta$ exactly like the partition function of an ensemble of massless particles. These results provide evidence to the effect that the qualitative ideas in [1] underlying the nuclearity condition are also applicable in theories with long-range forces.

\section{Nuclear Sets in Fock Space}

In this section we analyze the nuclearity properties of certain specific sets in Fock space. These general results will be applied to concrete models in the two subsequent sections.

Let $\mathscr{K}$ be a separable Hilbert space (which will be identified with the space of single-particle wavefunctions). The elements of $\mathscr{K}$ are denoted by $f, g$ and the scalar product in $\mathscr{K}$ by $\langle. \mid$.$\rangle . We also distinguish some anti-unitary involution J$ on $\mathscr{K}$ (which will be the operator of complex conjugation of the wavefunctions in configuration space). Given this structure one obtains by a standard construction the (symmetric) Fock space $\mathscr{H}$ over $\mathscr{K}$ with the scalar product $(.,$.$) and a distinguished vector \Omega$ representing the no-particle state (vacuum).

On $\mathscr{H}$ there act creation and annihilation operators $a^{*}(f)$ and $a(f)$, which are linear and anti-linear, respectively, in $f \in \mathscr{K}$, and which satisfy the usual canonical commutation relations. For the corresponding unitary Weyl operators given by

$$
W(f)=\exp \left(i\left(a^{*}(f)+a(f)\right)^{-}\right)
$$

one then has the well-known law of composition

$$
W(f) W(g)=W(f+g) \cdot \exp ((\langle g \mid f\rangle-\langle f \mid g\rangle) / 2) .
$$


We also consider the auxiliary field operators

$$
\varphi(f)=\left(a^{*}(f)+a(J f)\right) / \sqrt{2}, \quad \pi(g)=i\left(a^{*}(g)-a(J g)\right) / \sqrt{2}
$$

(which are linked to the Cauchy data of the local fields in the respective models). This familiar framework is supplemented by the following additional structure.

Firstly, we assume that we are given two closed subspaces $\mathscr{L}_{\varphi}$ and $\mathscr{L}_{\pi}$ of $\mathscr{K}$ which are invariant under the action of $J$. We then consider the real linear subspace $\mathscr{L}$ of $\mathscr{K}$ defined by

$$
\mathscr{L}=(1+J) \mathscr{L}_{\varphi}+(1-J) \mathscr{L}_{\pi}
$$

and the corresponding von Neumann algebra $\mathfrak{A}(\mathscr{L})$ which is generated by the Weyl operators $W(f)$ with $f \in \mathscr{L}$, i.e.,

$$
\mathfrak{U}(\mathscr{L})=\{W(f): f \in \mathscr{L}\}^{\prime \prime} .
$$

This algebra coincides with the von Neumann algebra generated by the field operators $\varphi(f), f \in \mathscr{L}_{\varphi}$ and $\pi(g), g \in \mathscr{L}_{\pi}$. (In our discussion of models we will choose the subspaces $\mathscr{L}_{\varphi}, \mathscr{L}_{\pi}$ in such a way that the corresponding algebra $\mathscr{U}(\mathscr{L})$ is the local algebra associated with a particular spacetime region.)

Secondly, we assume that we are given some unitary group $\mathbb{R} \ni t \rightarrow \mathrm{e}^{i t \gamma}$ on $\mathscr{K}$ whose generator $\gamma$ commutes with $J$. By a standard tensor-product construction we then obtain a unitary group $\mathbb{R} \ni t \rightarrow \mathrm{e}^{\mathrm{it} G}$ on $\mathscr{H}$ which leaves $\Omega$ invariant and satisfies

$$
\mathrm{e}^{i t G} W(f) \mathrm{e}^{-i t G}=W\left(\mathrm{e}^{i t \gamma} f\right) .
$$

(The generator $G$ will be the Hamiltonian in the models discussed below.)

We are interested in the question under which conditions on the spaces $\mathscr{L}_{\varphi}, \mathscr{L}_{\pi}$ and the generator $\gamma$ it holds true that

$$
\mathscr{M}(\gamma ; \mathscr{L})=\left\{\mathrm{e}^{-G} A \Omega: A \in \mathfrak{U}(\mathscr{L}),\|A\| \leqslant 1\right\}
$$

is a nuclear set of vectors in $\mathscr{H}$. Denoting by $E_{\varphi}$ and $E_{\pi}$ the orthogonal projections in $\mathscr{K}$ onto the subspaces $\mathscr{L}_{\varphi}$ and $\mathscr{L}_{\pi}$, respectively, for our purposes, a completely sufficient answer is given by the following

THEOREM 2.1. Let $E_{\varphi}, E_{\pi}$, and $\gamma$ be the projections and the generator, respectively, defined above and assume that $E_{\varphi} \cdot \mathrm{e}^{-\gamma}$ and $E_{\pi} \cdot \mathrm{e}^{-\gamma}$ are bounded operators of norm less than 1, which are in the trace class. Then $\mathscr{M}(\gamma ; \mathscr{L})$ is a nuclear set of vectors in $\mathscr{H}$ and $\star$

$$
v(\mathscr{M}(\gamma ; \mathscr{L})) \leqslant \operatorname{det}\left(1-\left|E_{\varphi} \cdot \mathrm{e}^{-\gamma}\right|\right)^{-2} \cdot \operatorname{det}\left(1-\left|E_{\pi} \cdot \mathrm{e}^{-\gamma}\right|\right)^{-2} .
$$

REMARK: We emphasize that this theorem applies also to unbounded operators $\mathrm{e}^{-\gamma}$. This is of interest in the analysis of modular operators in free field theory [6].

* Some notation: the norm, trace-norm, and Hilbert-Schmidt norm of an operator $A$ will be denoted by $\|A\|,\|A\|_{1}$, and $\|A\|_{2}$, respectively, and its determinant by $\operatorname{det} A$. The modulus $|A|$ of $A$ is the operator defined by $|A|=\sqrt{A^{*} A}$. 
The proof of this theorem is similar to the arguments given in the Appendix of [1]; but we have to deal here with the possibility that the operator $e^{-\gamma}$ is unbounded. For that reason we consider first the subalgebra $\mathfrak{x}_{0}(\mathscr{L})$ of $\mathfrak{A}(\mathscr{L})$, consisting of all finite linear combinations of the Weyl operators $W(f), f \in \mathscr{L}$, which is weakly dense in $\mathscr{2}(\mathscr{L})$.

Since $E_{\varphi} \cdot \mathrm{e}^{-\gamma}$ and $E_{\pi} \cdot \mathrm{e}^{-\gamma}$ are bounded operators it follows by a simple calculation that the vectors $W(f) \Omega, f \in \mathscr{L}$ are in the domain of $\mathrm{e}^{-G}$, and consequently also their linear span $\mathfrak{x}_{0}(\mathscr{L}) \Omega$. We want to show that the closure of the set of vectors

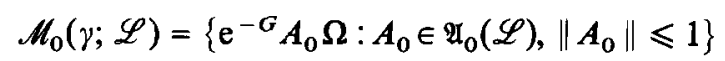

is nuclear. To this end we introduce a generating functional $\mathscr{G}$ given by (cf. [1])

$$
\mathscr{G}\left(f ; \mathrm{e}^{-G} A_{0} \Omega\right)=\left(\exp \left(a^{*}(f)\right) \cdot \Omega, \mathrm{e}^{-G} A_{0} \Omega\right),
$$

where $f \in \mathscr{K}, A_{0} \in \mathfrak{A}_{0}(\mathscr{L})$ and the exponential of the creation operator is defined in terms of its power-series expansion. Restricting $f$ to the domain of $\mathrm{e}^{-\gamma}$ we have the trivial identity

$$
\mathscr{G}\left(f, \mathrm{e}^{-G} A_{0} \Omega\right)=\mathscr{G}\left(\mathrm{e}^{-\gamma} f ; A_{0} \Omega\right)
$$

and to the right-hand side we can apply the arguments in [1], p. 339, giving the bound

$$
\left|\mathscr{G}\left(f ; \mathrm{e}^{-G} A_{0} \Omega\right)\right| \leqslant \exp \left(\frac{1}{2}\left\|\mathrm{e}^{-\gamma} f-g\right\|^{2}\right) \cdot\left\|A_{0}\right\| \text {. }
$$

Here $g$ is any element in the symplectic complement $\mathscr{L}^{\prime}$ of $\mathscr{L}$,

$$
\mathscr{L}^{\prime}=\{g \in \mathscr{K}:\langle g \mid f\rangle=\langle f \mid g\rangle \text { for all } f \in \mathscr{L}\} .
$$

Setting in the above inequality

$$
g=\frac{1}{2}\left(1-E_{\varphi}\right)(1-J) \mathrm{e}^{-\gamma} f+\frac{1}{2}\left(1-E_{\pi}\right)(1+J) \mathrm{e}^{-\gamma} f
$$

(which is easily seen to be an element of $\mathscr{L}^{\prime}$ if one takes into account that the projections $E_{\varphi}, \mathrm{E}_{\pi}$ commute with $J$ ) we arrive at

$$
\left|\mathscr{G}\left(f ; \mathrm{e}^{-G} A_{0} \Omega\right)\right| \leqslant \exp \left[\frac{1}{8}\left\|T_{\varphi}(1-J) f\right\|^{2}+\frac{1}{8}\left\|T_{\pi}(1+J) f\right\|^{2}\right] \cdot\left\|A_{0}\right\|,
$$

where we have put

$$
T_{\varphi}=\left|E_{\varphi} \cdot \mathrm{e}^{-\gamma}\right| \text { and } T_{\pi}=\left|E_{\pi} \cdot \mathrm{e}^{-\gamma}\right| .
$$

This estimate extends by continuity to all $f \in \mathscr{K}$ since the functional $f \rightarrow \mathscr{G}\left(f ; \mathrm{e}^{-G} A_{0} \Omega\right)$ is continuous on $\mathscr{K}$ (equipped with the strong topology), and the operators $T_{\varphi}, T_{\pi}$ are bounded.

The latter bound on the functional $\mathscr{G}$ can be simplified with the help of an operator $T$ which, in some sense, is the least upper bound of the operators $T_{\varphi}$ and $T_{\pi}$ : it is the smallest operator for which

$$
T^{n} \geqslant T_{\varphi}^{n} \quad \text { and } \quad T^{n} \geqslant T_{\pi}^{n}, \quad n \in \mathbb{N} .
$$


We will need the facts that

$$
\|T\| \leqslant \max \left(\left\|T_{\varphi}\right\|,\left\|T_{\pi}\right\|\right)
$$

and

$$
\left\|T^{n}\right\|_{1} \leqslant\left\|T_{\varphi}^{n}\right\|_{1}+\left\|T_{\pi}^{n}\right\|_{1}, \quad n \in \mathbb{N} .
$$

Since we did not find a reference for these statements, we briefly indicate how $T$ can be constructed: setting

$$
S_{m}=\left(\frac{1}{2}\left[T_{\varphi}^{2^{m}}+T_{\pi}^{2^{m}}\right]\right)^{2^{-m}}, \quad m \in \mathbb{N}
$$

one obtains a sequence of positive operators which satisfy $\left\|S_{m}\right\| \leqslant$ $\max \left(\left\|T_{\varphi}\right\|,\left\|T_{\pi}\right\|\right)$. It is easily seen that $\left(S_{m+1}\right)^{2^{m+1}} \geqslant\left(S_{m}\right)^{2^{m+1}}$ and making use of the fact that the function $x \rightarrow x^{c}, x \geqslant 0$ is operator monotonous for any $0<c \leqslant 1$ it follows that $S_{m+1} \geqslant S_{m}$. Hence, the sequence $S_{m}, m \in \mathbb{N}$ is increasing and bounded and therefore converges strongly to some operator $T$.

It is obvious from this construction that $T$ has the properties (2.16) and (2.17). Moreover, since for any $0<\mathrm{c} \leqslant 1$ and any pair of positive operators $A, B$ such that $A^{c}$ and $B^{c}$ are in the trace class one has

$$
\left\|(A+B)^{c}\right\|_{1} \leqslant\left\|A^{c}\right\|_{1}+\left\|B^{c}\right\|_{1}
$$

(cf., for example, [7]), it is also straightforward to establish (2.18). (That $T$ is the smallest operator with these properties is an immediate consequence of the fact that any operator $T^{\prime}$ for which (2.16) holds satisfies $T^{\prime} \geqslant S_{m}$ for $m \in \mathbb{N}$.)

Turning back to our estimate of the functional $\mathscr{G}$, we can proceed now from the previous bound on $\mathscr{G}$ to

$$
\mathscr{G}\left(f ; \mathrm{e}^{-G} A_{0} \Omega\right) \leqslant \mathrm{e}^{\frac{1}{2}\|T f\|^{2}} \cdot\left\|A_{0}\right\|,
$$

where we made use of relation (2.16) for $n=2$ and the fact that $T$ commutes with $J$ (since $T_{\varphi}$ and $T_{\pi}$ do). From (2.17), (2.18) and the assumed properties of $E_{\varphi}, E_{\pi}$, and $\gamma$, it is also clear that $\|T\|<1$ and $\|T\|_{1}<\infty$. Hence, applying the arguments given in the proof of Lemma 3 in the Appendix of [1], we find that there exists an orthonormal basis $\Phi_{n} \in \mathscr{H}, n \in \mathbb{N}$ such that

$$
\sum_{n=1}^{\infty} \sup \left\{\left|\left(\Phi_{n}, \Psi\right)\right|: \Psi \in \mathscr{M}_{0}(\gamma ; \mathscr{L})\right\} \leqslant \operatorname{det}(1-T)^{-2}<\infty
$$

This relation obviously stays true if we replace the set $\mathscr{M}_{0}(\gamma ; \mathscr{L})$ by its weak closure $\overline{\mathscr{M}_{0}(\gamma ; \mathscr{L})}$. Since the vectors $\Phi_{n}$ form an orthonormal basis, we also have $\sum_{n=1}^{\infty}\left(\Phi_{n}, \Psi\right) \cdot \Phi_{n}=\Psi$ for any $\Psi \in \mathscr{H}$. Hence we conclude that $\overline{\mathscr{M}_{0}(\gamma ; \mathscr{L})}$ is a nuclear set and that its nuclearity index is bounded from above by $\operatorname{det}(1-T)^{-2}$.

With this information it is easy to show that the set $\mathscr{M}(\gamma ; \mathscr{L})$ is nuclear too: since $\mathscr{A}_{0}(\mathscr{L})$ is weakly dense in $\mathfrak{A}(\mathscr{L})$ there exists, by Kaplansky's density theorem for each operator $A \in \mathfrak{A}(\mathscr{L})$ with $\|A\| \leqslant 1$, a sequence of operators $A_{n} \in \mathfrak{A}_{0}(\mathscr{L})$ with $\left\|A_{n}\right\| \leqslant 1$ such that $w-\lim A_{n} \Omega=A \Omega$. Moreover, the sequence $\mathrm{e}^{-G} A_{n} \Omega$, being contained in the 
nuclear set $\mathscr{M}_{0}(\gamma ; \mathscr{L})$, is bounded and therefore contains weakly convergent subsequences. But this implies that the vector $A \Omega$ is in the domain of $\mathrm{e}^{-G}$ since this operator

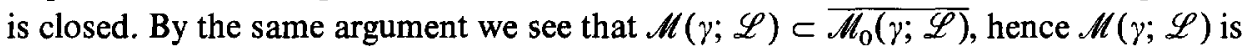
a nuclear set and

$$
v(\mathscr{M}(\gamma ; \mathscr{L})) \leqslant \operatorname{det}(1-T)^{-2} .
$$

It remains to proceed from this estimate to the bound given in the theorem. This is easily accomplished if one uses the equality

$$
\operatorname{det}(1-T)^{-2}=\exp \left(2 \cdot \sum_{n=1}^{\infty} \frac{1}{n}\left\|T^{n}\right\|_{1}\right)
$$

which holds for any positive trace-class operator $T$ with $\|T\|<1$. Applying to the right-hand side of this relation the estimate (2.18) for our particular operator $T$, we obtain

$$
\operatorname{det}(1-T)^{-2} \leqslant \operatorname{det}\left(1-T_{\varphi}\right)^{-2} \cdot \operatorname{det}\left(1-T_{\pi}\right)^{-2}
$$

which completes our proof of the theorem.

We conclude this section with an auxiliary lemma which simplifies the evaluation of the determinants appearing in the theorem.

LEMMA 2.2. Let $E$ be any projection. Then

$$
\operatorname{det}\left(1-\left|E \mathrm{e}^{-\gamma}\right|\right)^{-2} \leqslant \exp \left(2 \cdot \sum_{n=1}^{\infty} \frac{1}{n}\left\|E \mathrm{e}^{-n \gamma}\right\|_{1}\right)
$$

provided the right-hand side of this inequality exists. (This condition also implies that $\left\|E \mathrm{e}^{-\gamma}\right\|<1$.)

Proof. We will show below that if the operators $E \cdot \mathrm{e}^{-n \gamma}, n \in \mathbb{N}$ are in the trace class, one has

$$
\left\|\left|E \cdot \mathrm{e}^{-\gamma}\right|^{n}\right\|_{1} \leqslant\left\|E \mathrm{e}^{-n \gamma}\right\|_{1} .
$$

Anticipating this result it is clear that $\left\|E \mathrm{e}^{-n \gamma}\right\|_{1} \geqslant\left\|E \mathrm{e}^{-\gamma}\right\|^{n}$, therefore the premises of the lemma imply that $\left\|E \mathrm{e}^{-\gamma}\right\|<1$. Thus we can represent the determinant by the expression given in (2.23), and the statement of the lemma then follows by applying the above inequality a second time. So it only remains to establish this inequality.

Let $e_{i}, i \in \mathbb{N}$ be the family of orthonormal eigenvectors corresponding to the nonzero eigenvalues $\varepsilon_{i}$ of the operator $\left|E \cdot \mathrm{e}^{-\gamma}\right|$. Since the operators $\mathrm{e}^{-n \gamma} \cdot\left|E \mathrm{e}^{-\gamma}\right|^{2}$ are bounded, it is clear that the vectors $e_{i}$ are in the domain of $\mathrm{e}^{-n \gamma}$ for any $n \in \mathbb{N}$. Furthermore, it can be shown that for any positive $m \in \mathbb{R}$

$$
\varepsilon_{i}^{m}=\left\langle\left. e_{i}|| E \mathrm{e}^{-\gamma}\right|^{m} e_{i}\right\rangle \leqslant\left\langle e_{i} \mid \mathrm{e}^{-m y} \cdot e_{i}\right\rangle .
$$

For $0<m \leqslant 2$, this is a consequence of

$$
\left|E \mathrm{e}^{-\gamma}\right|^{2}=\mathrm{e}^{-\gamma} E \mathrm{e}^{-\gamma} \leqslant \mathrm{e}^{-2 \gamma}
$$


and the fact (already mentioned) that the function $x \rightarrow x^{c}, x \geqslant 0$ is operator monotonous if $0<c \leqslant 1$. Taking into account that $e_{i}$ is a normalized eigenvector of $\left|E \mathrm{e}^{-\gamma}\right|$, the statement then follows for all $m>0$ since if $M$ is a sufficiently large power of 2 (such that $m / M \leqslant 2$ ) one has

$$
\begin{aligned}
\varepsilon_{i}^{m}=\left(\varepsilon_{i}^{m / M}\right)^{M} & \leqslant\left\langle e_{i} \mid \mathrm{e}^{-(m / M) \gamma} \cdot e_{i}\right\rangle^{M} \\
& \leqslant e_{i}\left|\mathrm{e}^{-m \gamma \cdot} \cdot e_{i}\right\rangle
\end{aligned}
$$

where the last step follows by repeated application of Cauchy-Schwarz inequality. Hence, if $n \in \mathbb{N}, n \geqslant 2$ one obtains

$$
\begin{aligned}
\left\|\left|E \mathrm{e}^{-\gamma}\right|^{n}\right\|_{1} & =\sum_{i=1}^{\infty} \varepsilon_{i}^{n} \leqslant \sum_{i=1}^{\infty} \varepsilon_{i}^{2}\left\langle e_{i} \mid \mathrm{e}^{-(n-2) \gamma} \cdot e_{i}\right\rangle \\
& =\sum_{i=1}^{\infty}\left\langle e_{i} \mid \mathrm{e}^{-\gamma} E \mathrm{e}^{-(n-1) \gamma} \cdot e_{i}\right\rangle=\operatorname{Tr} \mathrm{e}^{-\gamma} E \mathrm{e}^{-(n-1) \gamma} \\
& =\operatorname{Tr} E \mathrm{e}^{-n \gamma} E \leqslant\left\|E \mathrm{e}^{-n \gamma}\right\|_{1} .
\end{aligned}
$$

This completes the proof of the lemma for $n \geqslant 2$. For $n=1$ the statement is trivial.

We are now in a position to establish the nuclearity condition in models of noninteracting massless particles.

\section{Free Massless Scalar Fields}

As a first example we consider the free field theory of a massless scalar particle. In this model the Hilbert space $\mathscr{K}$ is identified with the space $L^{2}\left(\mathbb{R}^{3}\right)$ of the single particle wave functions $f(\mathbf{x}), \mathbf{x} \in \mathbb{R}^{3}$ in configuration space. The anti-unitary involution $J$ is given by

$$
(J f)(\mathbf{x})=\overline{f(\mathbf{x})},
$$

and the generator $\omega$ of the time translations $\mathbb{R} \ni t \rightarrow \mathrm{e}^{i t \omega}$ on $\mathscr{K}$ is defined by

$$
(\tilde{\omega} f)(\mathbf{p})=|\mathbf{p}| \cdot \tilde{f}(\mathbf{p}) \text {, }
$$

where the tilde denotes Fourier transformation. Since the function $\mathbf{p} \rightarrow|\mathbf{p}|$ is real and symmetric, $\omega$ commutes with $J$. The generator of the corresponding time translation in $\mathscr{H}$ (the Hamiltonian) will be denoted by $H$.

The underlying local scalar field $\phi$ is related to the auxiliary fields $\varphi, \pi$ introduced in the previous section through its Cauchy data at time $t=0$. Namely if $f, g$ are test functions on $\mathbb{R}^{3}$ one has

$$
\phi(t=0, f)=\varphi\left(\omega^{-1 / 2} f\right), \quad \phi(t=0, g)=\pi\left(\omega^{1 / 2} g\right),
$$

where on the right-hand side of these equations we have identified the functions $f, g$ with the corresponding elements of $\mathscr{K}$.

Now let $r>0$ and let $\mathbf{O}_{r}=\left\{\mathbf{x} \in \mathbb{R}^{3}:|\mathbf{x}| \leqslant r\right\}$. We distinguish two subspaces of $\mathscr{K}$,

$$
\mathscr{L}_{\varphi}(r)=\overline{\omega^{-1 / 2} \mathscr{D}\left(\mathbf{O}_{r}\right)} \text { and } \mathscr{L}_{\pi}(r)=\overline{\omega^{1 / 2} \mathscr{D}\left(\mathbf{O}_{r}\right)} \text {, }
$$


where $\mathscr{D}\left(\mathbf{O}_{r}\right)$ denotes the set of all complex valued test functions with support in $\mathbf{O}_{r}$, and the bar indicates closure in $\mathscr{K}$. Both spaces are clearly invariant under $J$. As was discussed in the previous section, there is an associated real linear space $\mathscr{L}(r) \subset \mathscr{K}$ (cf. definition (2.4)) and a corresponding von Neumann algebra $\mathfrak{U}(\mathscr{L}(r))$ acting on $\mathscr{H}$. It is obvious from the relation between the auxiliary fields $\varphi, \pi$ and the local field $\phi$ that this algebra is precisely the local algebra which is attached to the double cone $\mathcal{O}_{r}$ with base $\mathbf{O}_{r}$ at time $t=0$. Hence, the sets $\mathcal{N}\left(\mathcal{O}_{r}, \beta\right)$ appearing in the formulation of the nuclearity condition are of the type considered in the previous section. With the help of those results we will establish

THEOREM 3.1. In the free field theory of a single spinless particle of mass 0 the sets

$$
\mathscr{N}\left(\mathcal{O}_{r}, \beta\right)=\left\{\mathrm{e}^{-\beta H} U \Omega: U \in \mathfrak{U}(\mathscr{L}(r)), U^{*} U=1\right\}
$$

are nuclear for any $r>0$ and $\beta>0$. Moreover, there exist constants $c, c^{\prime}$ such that

$$
v\left(\mathcal{N}\left(\mathcal{O}_{r}, \beta\right)\right) \leqslant \begin{cases}\mathrm{e}^{c(r / \beta)^{3}} & \text { for } r \geqslant \beta, \\ \mathrm{e}^{c^{\prime}(r / \beta)} & \text { for } r<\beta .\end{cases}
$$

For the proof of this statement we must analyze the properties of the operators $E_{\varphi}(r) \mathrm{e}^{-\beta \omega}$ and $E_{\pi}(r) \mathrm{e}^{-\beta \omega}$ on $\mathscr{K}$, where $E_{\varphi}(r)$ and $E_{\pi}(r)$ are the projections onto the subspaces $\mathscr{L}_{\varphi}(r)$ and $\mathscr{L}_{\pi}(r)$, respectively. To this end we introduce on $\mathscr{K}$ an operator $\chi_{r}$ given by

$$
\left(\chi_{r} f\right)(\mathbf{x})=\chi_{1}(\mathbf{x} / r) \cdot f(\mathbf{x}),
$$

where $\chi_{1}$ is any test function satisfying $\chi_{1}(y)=1$ for $|\mathbf{y}| \leqslant 1$. Next we make use of the trivial identity (cf. [1])

$$
E \varphi(r) \cdot \omega^{1 / 2} \chi_{r} \omega^{-1 / 2}=E_{\varphi}(r)
$$

and decompose the operator $E_{\varphi}(r) \mathrm{e}^{-\beta \omega}$ into the product

$$
E_{\varphi}(r) \cdot\left\{\omega^{1 / 2} \chi_{r} \omega^{-1 / 2} \cdot\left(1+\lambda^{2} \omega^{2}\right)^{-2}\right\} \cdot\left\{\left(1+\lambda^{2} \omega^{2}\right)^{2} \omega^{1 / 2} \chi_{r} \omega^{-1 / 2} \mathrm{e}^{-\beta \omega}\right\}
$$

where $\lambda=\min (r, \beta)$. By inspection of the kernels (in momentum space) of the operators in the curly brackets, it is easily seen that they are in the Hilbert-Schmidt class. Moreover, by a straightforward estimate of their respective Hilbert-Schmidt norms, one obtains the bounds

$$
\begin{aligned}
& \left\|\omega^{1 / 2} \chi_{r} \omega^{-1 / 2}\left(1+\lambda^{2} \omega^{2}\right)^{-2}\right\|_{2} \leqslant c \cdot\left(1+(r / \beta)^{3}\right)^{1 / 2}, \\
& \left\|\left(1+\lambda^{2} \omega^{2}\right)^{2} \omega^{1 / 2} \chi_{r} \omega^{-1 / 2} \mathrm{e}^{-\beta \omega}\right\|_{2} \leqslant c \cdot\left((r / \beta)^{2}+(r / \beta)^{3}\right)^{1 / 2} .
\end{aligned}
$$

(Here and in the following, the letter $c$ stands for certain numerical constants.) Thus the operator $E_{\varphi}(r) \mathrm{e}^{-\beta \omega}$ is in the trace class, and its trace norm, being smaller than the product of the preceding two Hilbert-Schmidt norms, can be estimated by

$$
\left\|E_{\varphi}(r) \mathrm{e}^{-\beta \omega}\right\|_{1} \leqslant c\left((r / \beta)+(r / \beta)^{3}\right) .
$$


By an analogous argument one finds that $E_{\pi}(r) \mathrm{e}^{-\beta \omega}$ is in the trace class too, and

$$
\left\|E_{\pi}(r) \mathrm{e}^{-\beta \omega}\right\|_{1} \leqslant c\left((r / \beta)^{2}+(r / \beta)^{3}\right) .
$$

Since in these estimates one can replace $\beta$ by $n \cdot \beta, n \in \mathbb{N}$, it is obvious that the conditions of Lemma 2.2 are satisfied so that we have

$$
\left\|E_{\varphi}(r) \mathrm{e}^{-\beta \omega}\right\|<1 \quad \text { and }\left\|E_{\pi}(r) \mathrm{e}^{-\beta \omega}\right\|<1 .
$$

(This bound can also be derived from the fact that the operators in question are compact and that the spectrum of $\omega$ is positive and continuous.) Moreover, we obtain from Lemma 2.2 the bounds

$$
\begin{aligned}
& \operatorname{det}\left(1-\left|E_{\varphi}(r) \mathrm{e}^{-\beta \omega}\right|\right)^{-2} \leqslant \mathrm{e}^{c\left((r / \beta)+(r / \beta)^{3}\right)} \\
& \operatorname{det}\left(1-\left|E_{\pi}(r) \mathrm{e}^{-\beta \omega}\right|\right)^{-2} \leqslant \mathrm{e}^{c\left((r / \beta)^{2}+(r / \beta)^{3}\right)} .
\end{aligned}
$$

Applying Theorem 2.1, we thus conclude that the sets

$$
\mathscr{M}(\beta \omega ; \mathscr{L}(r))=\left\{\mathrm{e}^{-\beta H} A \Omega: A \in \mathfrak{U}(\mathscr{L}(r)),\|A\| \leqslant 1\right\}
$$

are nuclear for any $r>0, \beta>0$ and that

$$
v\left(\mathscr{M}(\beta \omega ; \mathscr{L}(r)) \leqslant \mathrm{e}^{c\left((r / \beta)+(r / \beta)^{3}\right)} .\right.
$$

Since the sets $\mathcal{N}\left(\mathcal{O}_{r}, \beta\right)$ we are interested in are subsets of $\mathscr{M}(\beta \omega ; \mathscr{L}(r))$ the statement of Theorem 3.1 now follows.

This argument can easily be extended to theories describing finite multiplets of scalar massless particles. There the single particle space is $\mathscr{K}=L^{2}\left(\mathbb{R}^{3}\right) \otimes \mathbb{C}^{f}$ where $f$ is the number of species of particles in the model. The corresponding anti-unitary involution, the Hamiltonian, and the projections onto the 'local' subspaces are obtained by taking tensor products of the operators $J, \omega$ and $E_{\varphi}(r), E_{\pi}(r)$, respectively, with the unit operator on $\mathbb{C}^{f}$. Theorem 3.1 applies to all these models if one replaces the numerical constants $c, c^{\prime}$ appearing in its formulation by $f \cdot c$ and $f \cdot c^{\prime}$, respectively.

\section{Free Electromagnetic Field}

As an example of more physical interest, we now treat the theory of the free electromagnetic field. It will be convenient to consider besides the physical ('transversal') photons also unphysical ('longitudinal') photons. The single particle space in this extended model is $\mathscr{K}=L^{2}\left(\mathbb{R}^{3}\right) \otimes \mathbb{C}^{3}$. In order to emphasize the 3-vector character of the elements of $\mathscr{K}$, we denote them in the following by bold-face letters $\mathbf{f}, \mathbf{g}$. The involution $J$ is given by

$$
(J \mathbf{f})(\mathbf{x})=\overline{\mathbf{f}(\mathbf{x})},
$$

and the generator $\omega$ of the time translations by

$$
(\tilde{\omega} \mathbf{f})(\mathbf{p})=|\mathbf{p}| \cdot \tilde{\mathbf{f}}(\mathbf{p}) \text {. }
$$


The resulting Fock space and the Hamiltonian are denoted by $\mathscr{H}$ and $H$, respectively.

The physical photons are described by vectors in the subspace $\mathscr{K}_{\mathrm{T}} \subset \mathscr{K}$

$$
\mathscr{K}_{T}=\{\mathbf{f} \in \mathscr{K}: \mathbf{p} \cdot \tilde{f}(\mathbf{p})=0 \text { a.e. }\} .
$$

We denote the corresponding Fock space (regarded as a subspace of $\mathscr{H}$ ) by $\mathscr{H}_{T}$. It is obvious that $\mathscr{H}_{T}$ is invariant under the action of the Hamiltonian $H$.

The electromagnetic fields $\mathbf{E}, \mathbf{H}$ are related to the auxiliary fields $\varphi, \pi$ as follows: if $\mathbf{f}, \mathbf{g}$ are (vector-valued) test functions on $\mathbb{R}^{3}$, one puts

$$
\begin{aligned}
& \mathbf{E}(t=0, \mathbf{f})=\pi\left(\omega^{1 / 2} P_{T} \mathbf{f}\right) \\
& \mathbf{H}(t=0, \mathbf{g})=\varphi\left(-\omega^{-1 / 2} \operatorname{curl} \mathbf{g}\right)
\end{aligned}
$$

where $P_{T}$ is the projection in $\mathscr{K}$ onto $\mathscr{K}_{T}$. With this identification $\mathbf{E}, \mathbf{H}$ satisfy the Maxwell equations and have the correct commutation relations. We also see from it how to define the 'local' subspaces of $\mathscr{K}$ corresponding to the double cone $\mathcal{O}_{r}$ with base $\mathbf{O}_{r}$ at time $t=0$ :

$$
\begin{aligned}
& \mathscr{L}_{\varphi}(r)=\overline{\omega^{-1 / 2} \cdot \operatorname{curl}\left(\mathscr{D}\left(\mathbf{O}_{r}\right) \otimes \mathbb{C}^{3}\right)}, \\
& \mathscr{L}_{\pi}(r)=\overline{\omega^{1 / 2} \cdot P_{T}\left(\mathscr{D}\left(\mathbf{O}_{r}\right) \otimes \mathbb{C}^{3}\right)} .
\end{aligned}
$$

These spaces are invariant under the action of $J$ and are contained in $\mathscr{K}_{T}$.

In the following we will show that the corresponding sets $\mathscr{M}(\beta \omega ; \mathscr{L}(r))$ of vectors satisfy the nuclearity condition with regard to $\mathscr{H}$. Since these sets are contained in the physical subspace $\mathscr{H}_{T}$ it is then clear that they also satisfy the nuclearity condition with regard to $\mathscr{H}_{T}$. Hence, we have

THEOREM 4.1. In the theory of the free electromagnetic field, the sets $\mathcal{N}\left(\mathcal{O}_{r}, \beta\right)$ are nuclear for any $r>0$ and $\beta>0$. Moreover,

$$
v\left(\mathscr{N}\left(\mathcal{O}_{r}, \beta\right)\right) \leqslant \begin{cases}\mathrm{e}^{c(r / \beta)^{3}}, & \text { for } r \geqslant \beta, \\ \mathrm{e}^{c^{\prime}(r / \beta)}, & \text { for } r<\beta\end{cases}
$$

where $c, c^{\prime}$ are constants.

For the proof of this statement we must study the spectral properties of the operators $E_{\varphi}(r) \mathrm{e}^{-\beta \omega}$ and $E_{\pi}(r) \mathrm{e}^{-\beta \omega}$ determined by $\mathscr{L}_{\varphi}(r), \mathscr{L}_{\pi}(r)$, and $\omega$. In fact, it suffices to analyze either one of these operators since they are unitarily equivalent. This may be seen as follows: let $V$ be the partial isometry on $\mathscr{K}$ given by

$$
(\tilde{V \mathbf{f}})(\mathbf{p})=i(\mathbf{p} \times \tilde{\mathbf{f}}(\mathbf{p})) /|\mathbf{p}| \text {. }
$$

One easily checks that the restriction of $V$ to $\mathscr{K}_{T}$ is selfadjoint and unitary, and $V E_{\varphi}(r) V=E_{\pi}(r)$. Since $V$ commutes with $\omega$, this implies that

$$
V E_{\varphi}(r) \mathrm{e}^{-\beta \omega} V=E_{\pi}(r) \mathrm{e}^{-\beta \omega},
$$

hence, the norms and trace norms of the two operators are equal. (Note that the equivalence of these operators is linked to the symmetry $(\mathbf{E}, \mathbf{H}) \rightarrow(\mathbf{H},-\mathbf{E})$ of the theory.) 
It remains to control the trace-norm of $E_{\varphi}(r) \mathrm{e}^{-\beta \omega}$. Denoting by $F_{\varphi}(r)$ the projection onto the subspace $\overline{\left\{\omega^{-1 / 2}\left(\mathscr{D}\left(\mathbf{O}_{r}\right) \otimes \mathbb{C}^{3}\right)\right\}}$ of $\mathscr{K}$ it is clear that $E_{\varphi}(r) \leqslant F_{\varphi}(r)$, and consequently $\left|E_{\varphi}(r) \mathrm{e}^{-\beta \omega}\right| \leqslant\left|F_{\varphi}(r) \mathrm{e}^{-\beta \omega}\right|$. The latter operator is exactly of the type considered in the previous section. Applying the arguments given there one finds that

$$
\left\|F_{\varphi}(r) \mathrm{e}^{-\beta \omega}\right\|_{1} \leqslant c \cdot\left((r / \beta)+(r / \beta)^{3}\right)
$$

and consequently

$$
\left\|E_{\varphi}(r) \mathrm{e}^{-\beta \omega}\right\|_{1}=\left\|E_{\pi}(r) \mathrm{e}^{-\beta \omega}\right\|_{1} \leqslant c\left((r / \beta)+(r / \beta)^{3}\right) .
$$

The statement of the theorem now follows from the results in Section 2.

\section{References}

1. Buchholz, D. and Wichmann, E. H., 'Causal Independence and the Energy-Level Density of States in Local Quantum Field Theory', Commun. Math. Phys. 106, 321 (1986).

2. Buchholz, D., D'Antoni, C., and Fredenhagen, K., 'The Universal Structure of Local Algebras', DESY-preprint 86-158.

3. D'Antoni, C., Doplicher, S., Fredenhagen, K., and Longo, R., 'Convergence of Local Charges and Continuity Properties of $W^{*}$-Inclusions', DESY-preprint 86-162.

4. Buchholz, D. and Junglas, P., 'Local Properties of Equilibrium States and the Particle Spectrum in Quantum Field Theory', Lett. Math. Phys. 11, 51 (1986).

5. Buchholz, D., 'On Particles, Infraparticles, and the Problem of Asymptotic Completeness', to appear in the proceedings of the IAMP-conference, Marseille, 1986.

6. Buchholz, D., D'Antoni, C., and Longo, R., work in progress.

7. Kosaki, H., 'On the Continuity of the Map $\varphi \rightarrow|\varphi|$ from the Predual of a $W^{*}$-Algebra', J. Funct. Anal. 59, 123 (1984). 